\title{
Influence of cobalt substitution on the structural and magnetic properties of cobalt substituted magnetite
}

\begin{abstract}
Chemical substitution is one of the methods to modify magnetic and structural properties of magnetic materials. Substitution of cobalt ions in magnetite lattice has been done via coprecipitation method to investigate the effect of cobalt substitution in altering the structural and magnetic properties of $\mathrm{Co}(\mathrm{x}) \mathrm{Fe}(1-\mathrm{x}) \mathrm{Fe} 2 \mathrm{O} 4$. Changes in the lattice constant have been observed with increase in cobalt concentration though particle size varied insignificantly. Qualitative study of the cation occupancy at tetrahedral and octahedral sites was observed at wavenumbers ranging from 600 to $400 \mathrm{~cm}-1$, known as the characteristic range for ferrites. Preferential occupancy of cobalt ions into tetrahedral sites was evident by the changes in the intensity of transmitted FTIR signal as the wavenumbers shifted towards longer wavenumber with increasing cobalt concentration. Meanwhile for saturation magnetization, only small changes is observed when $\mathrm{Fe} 2+$ is completely substituted by Co2+. Increment of $21.3 \%$ in the saturation magnetization of $1.0 \mathrm{Co}$ was achieved in comparison with pure magnetite, therefore proves the role of cation substitution in the enhancement of saturation magnetization.
\end{abstract}

Keyword: Cobalt substitution; Magnetic; $\mathrm{Co}(\mathrm{x}) \mathrm{Fe}(1-\mathrm{x}) \mathrm{Fe} 2 \mathrm{O} 4$; Magnetization 